Proceedings

\title{
Locating the QCD Critical Point Using Holographic Black Holes ${ }^{\dagger}$
}

\author{
Israel Portillo \\ University of Houston, Department of Physics, Houston, TX 77004, USA; iportillovazquez@gmail.com \\ † Presented at Hot Quarks 2018-Workshop for Young Scientists on the Physics of Ultrarelativistic \\ Nucleus-Nucleus Collisions, Texel, The Netherlands, 7-14 September 2018.
}

Published: 29 April 2019

\begin{abstract}
It has been shown that holographic black holes, constructed to mimic the equation of state of QCD computed on the lattice at finite temperature and zero density, display critical behavior at large baryonic chemical potential. In this proceedings, we discuss the mapping of holographic black holes into the QCD phase diagram and the emergence of the critical point and the first order phase transition line.
\end{abstract}

Keywords: QCD phase diagram; critical point; holography; black hole engineering; quark gluon plasma

\section{Introduction}

The study of the non perturbative QCD phenomena at finite temperature $(T)$ has been possible over the last years through calculations performed on the lattice. Due to the well known sign problem, those calculations can only be done at zero (or imaginary) baryonic chemical potential $\left(\mu_{B}\right)$. The extension of those calculations to finite $\mu_{B}$ is currently limited to values of $\mu_{B} / T<2[1,2]$. In this density regime, lattice QCD has found a rapid crossover transition between a gas of hadrons and a deconfined quark gluon plasma phase [3,4]. Whether or not this crossover evolves into a critical point where a first order phase transition begins is an important question that cannot be addressed at the moment by first principle calculations. Nevertheless, the existence and location of the critical point are of considerable interest to experiments probing unprecedented high values of $\mu_{B}$ where the critical point may be found, especially to the second run of the Beam Energy Scan at BNL, and to the future Facility for Antiproton and Ion Research at GSI.

In the absence of first principle calculations for higher values of $\mu_{B}$, alternative approaches should be explored if one wants to relate heavy ion experiments to the phases of QCD and the critical point. One of those approaches is the holographic duality introduced in Ref. [5]. This method was used in Ref. [6] to construct five-dimensional black holes solutions with thermodynamic properties that mimic the equation of state calculated on the lattice at $\mu_{B}=0$. The model was extended to include a baryonic charge in Refs. $[7,8]$ where it was shown that such holographic constructions can display a critical point at large $\mu_{B}$. Having a non zero bulk viscosity, these non-conformal models play an important role in hydrodynamic simulations, and they have been used to compute high order baryonic susceptibilities [9-11], and transport coefficients [12-15].

Following our work in Ref. [9], in this contribution we discuss how the holographic black hole solutions map into the QCD phase diagram and produce a critical point and a first order phase transition. 


\section{Black Hole Model}

The action of the holographic model is given by $[7,8]$

$$
\mathcal{S}=\frac{1}{16 \pi G_{5}} \int \mathrm{d} x^{5} \sqrt{-g}\left[\mathcal{R}-\frac{1}{2}\left(\partial_{M} \phi\right)^{2}-V(\phi)-\frac{1}{4} f(\phi) F_{M N}^{2}\right] .
$$

where $G_{5}$ is the gravitational coupling, $\mathcal{R}$ is the Ricci scalar, and $\phi$ is a dilaton field which couple to the metric $g_{\mu v}$, and to a Maxwell dilaton field $A_{N}$ defining an Einstein-Maxwell-dilaton model (EMD). The conserved baryonic charge is taken into account by including the vector field $A_{\mathrm{N}}$ in the action in the bulk. The non-conformal behavior of the model is controlled by a scalar potential $V(\phi)$, and the coupling between $A_{\mathrm{N}}$ and $\phi$ by the function $f(\phi)$. Those two functions are engineered to reproduce the entropy density and the second baryon susceptibility at $\mu_{B}=0$ from the lattice, respectively (see Ref. [9] for details).

The charged black hole backgrounds, spatially isotropic and translationally invariant, that we are considering can be described by the following ansatz for the EMD fields [7]

$$
d s^{2}=e^{2 A(r)}\left[-h(r) d t^{2}+d \vec{x}^{2}\right]+\frac{e^{2 B(r)} d r^{2}}{h(r)} .
$$

The EMD model produces four coupled second order differential equations of motion given in terms of the fields $\{A, h, \phi, \Phi\}$, where $A=A(r), h=h(r), \phi=\phi(r)$, and $A=\Phi(r) d t$ depend only on the holographic variable $r$. A black hole solution may be obtained by integrating the equations of motion from a near-horizon to a far-horizon where, according to the holographic dictionary, the thermodynamics of the 4-dimensional gauge theory is computed. Therefore, to solve the equations of motion, it is necessary to define a boundary condition at the near-horizon of the EMD fields. As done in Ref. [7-9], this boundary is parametrized in terms of two initial values $\left(\phi_{0}, \Phi_{1}\right)$, which define the black hole solution. In the next session, we discuss how these two initial conditions translate into a point in the QCD phase diagram.

\section{Discussion}

The two initial conditions defining a holographic black hole are the value of the field at the near-horizon, $\phi_{0}$, and the value of the electric field, $\Phi_{1}$, in the holographic direction $r$. Therefore, $\Phi_{1}=0$ corresponds to the $T$ axis $\left(\mu_{B}=0\right)$ as shown on the left panel of Figure 1 . To populate the QCD phase diagram with black hole solutions, we fix $\phi_{0}$ to produce equally spaced intervals of $T$. Then, we vary $\Phi_{1}$ from zero to the maximum bound imposed by requiring an asymptotically AdS black hole, $\Phi_{1(\max )}=\sqrt{-2 V\left(\phi_{0}\right) / f\left(\phi_{0}\right)}$ [7]. The right panel in Figure 1 shows the lines of constant $\phi_{0}$ bending down as they evolve in the $\mu_{B}$ direction. We can distinguish between three kinds of lines: the dotted lines that do not cross each other; the dashed lines in the middle of the plane that cross some of the dotted lines; and the solid lines on the top of the plane that cross some of the dotted and dashed lines.
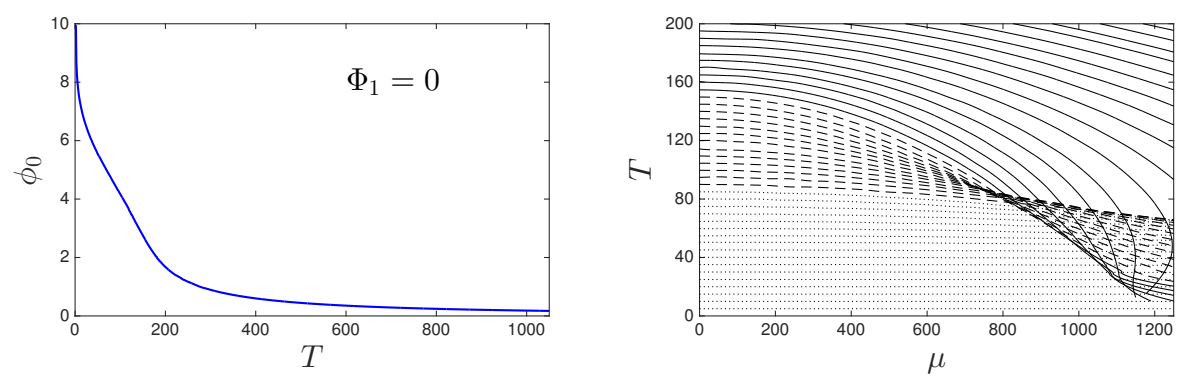

Figure 1. Mapping of the initial values of the black holes $\left(\phi_{0}, \Phi_{1}\right)$ to the QCD phase diagram $\left(T, \mu_{B}\right)$. The left panel shows the dependence on $T$ of $\phi_{0}$ when $\Phi_{1}=0$. The right panel shows the lines of constant $\phi_{0}$ evolving in the $\mu_{B}$ direction as $\Phi_{1}$ increases. 
The crossing of the lines creates a region of the phase diagram with multi-solutions that begins on the point $\left(T^{c}=89 \mathrm{MeV}, \mu_{B}^{c}=724 \mathrm{MeV}\right)$, and extends to lower $T$ and higher $\mu_{B}$. The stable solution in this region is required to minimize the free energy (or maximize the entropy), and also to be locally stable under small fluctuations. Thermodynamic stability requires the entropy $(S)$, baryon number $(N)$, specific heat at constant volume $\left(C_{v}=T(\partial S / \partial T)_{v}\right)$, and the quark susceptibility $\left(\chi_{2}=\left(\partial N / \partial \mu_{B}\right)_{T}\right)$, to be positive definite. Figure 2 shows $S$ and $N$ as functions of $T$ for three different values of $\mu_{B}$ : one below $\mu_{B}^{c}\left(\mu_{B}=700\right)$ and two above $\mu_{B}^{c}\left(\mu_{B}=750\right.$ and $\left.\mu_{B}=800\right)$. This figure also shows, with a thick red line, the values of $S$ and $N$ that are thermodynamically unstable. The unstable regions belong to the dashed lines in the phase diagram in Figure 1, which is the plane that is bent in the middle between the dotted and the solid lines. We also notice that $S$ is higher in the dotted lines than in the dashed lines at fixed $\mu_{B}$. Therefore, from the two remaining solutions, the one that maximizes the entropy will be the one with higher value of $\phi_{0}$.
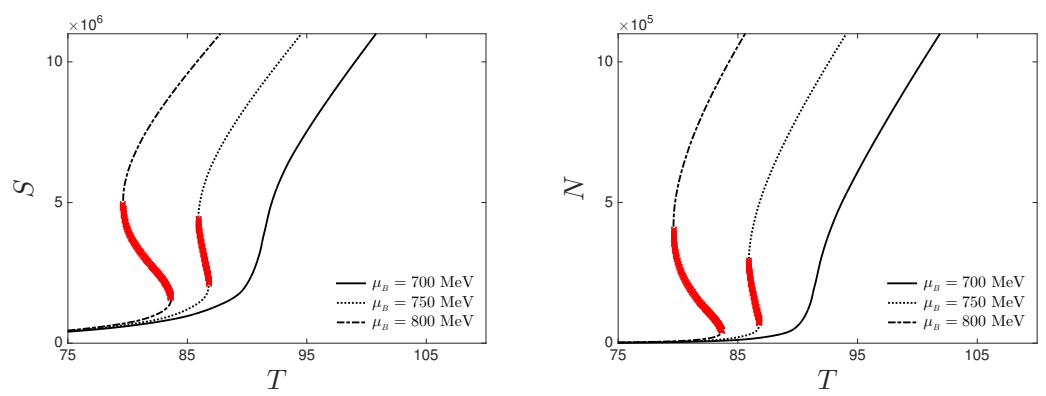

Figure 2. Entropy (left) panel and Baryon number (right) panel as functions of $T$ for $\mu_{B}=700 \mathrm{MeV}$, $\mu_{B}=750 \mathrm{MeV}$, and $\mu_{B}=800 \mathrm{MeV}$. The thick red lines indicate thermodynamically unstable points.

\section{Conclusions}

We used a holographic approach to construct black hole solutions parametrized by two initial values $\left(\phi_{0}, \Phi_{1}\right)$, and discuss how the black hole solutions map into the QCD phase diagram. We showed that the lines of constant $\phi_{0}$ bend to lower values of $T$ as $\Phi_{1}$ increases. The bending of the lines produces an overlap of black hole solutions. The overlapping region begins at the point $\left(T^{c}=89 \mathrm{MeV}, \mu_{B}^{c}=724 \mathrm{MeV}\right)$ and extends to lower values of $T$ and higher values of $\mu_{B}$ forming a bent plane with three different black hole solutions for a single $\left(T, \mu_{B}\right)$ point. In this region, we found that the solution with the intermediate value of $\phi_{0}$ (dashed lines on the right panel in Figure 1) is thermodynamically unstable. From the remaining two solutions, the one with higher $\phi_{0}$ has higher entropy. Therefore, the system will maximize the entropy by moving from the lower plane (dotted lines on the right panel in Figure 1) to the higher plane (solid lines on the right panel in Figure 1) when those two planes overlap developing a first order phase transition line along the boundary of those two regions. The point where the overlapping of the planes begins $\left(T^{c}=89 \mathrm{MeV}, \mu_{B}^{c}=724 \mathrm{MeV}\right)$ will identify the location of the QCD critical point in the holographic model.

Funding: This material is based upon work supported by the National Science Foundation under grant PHY-1654219 and OAC-1531814, and by the U.S. Department of Energy, Office of Science, Office of Nuclear Physics, within the framework of the Beam Energy Scan Theory (BEST) Topical Collaboration.

Acknowledgments: I would like to thank the organizers of the Hot Quarks 2018 conference, and my collaborators on this work: Renato Critelli, Jorge Noronha, Jacquelyn Noronha-Hostler, Claudia Ratti and Romulo Rougemont. I gratefully acknowledge the use of the Maxwell Cluster and the support from the Center of Advanced Computing and Data Systems at the University of Houston

Conflicts of Interest: The authors declare no conflict of interest. The founding sponsors had no role in the design of the study; in the collection, analyses, or interpretation of data; in the writing of the manuscript, or in the decision to publish the results. 


\section{References}

1. Ratti, C. Lattice QCD and heavy ion collisions: A review of recent progress. Rept. Prog. Phys. 2018, 81, 084301.

2. Bazavov, A.; Ding, H.T.; Hegde, P.; Kaczmarek, O.; Karsch, F.; Laermann, E.; Maezawa, Y.; Mukherjee, S.; Ohno, H.; Petreczky, P.; et al. QCD equation of state to $\mathcal{O} \mu_{B}^{6}$ from lattice QCD. Phys. Rev. D 2017, 95, 054504.

3. Aoki, Y.; Endrodi, G.; Fodor, Z.; Katz, S.D.; Szabo, K.K. The order of the quantum chromodynamics transition predicted by the standard model of particle physics. Nature 2006, 443, 675-678.

4. Bellwied, R.; Borsanyi, S.; Fodor, Z.; Günther, J.; Katz, S.D.; Ratti, C.; Szabo, K.K. The QCD phase diagram from analytic continuation. Phys. Lett. B 2015, 751, 559-564.

5. Maldacena, J.M. The large-N limit of superconformal field theories and supergravity. Int. J. Theor. Phys. 1999, $38,1113-1133$.

6. Gubser, S.S.; Nellore, A. Mimicking the QCD equation of state with a dual black hole. Phys. Rev. D 2008, $78,086007$.

7. DeWolfe, O.; Gubser, S.S.; Rosen, C. A holographic critical point. Phys. Rev. D 2011, 83, 086005.

8. DeWolfe, O.; Gubser, S.S.; Rosen, C. Dynamic critical phenomena at a holographic critical point. Phys. Rev. D 2011, 84, 126014.

9. Critelli, R.; Noronha, J.; Noronha-Hostler, J.; Portillo, I.; Ratti, C.; Rougemont, R. Critical point in the phase diagram of primordial quark-gluon matter from black hole physics. Phys. Rev. D 2017, 96, 096026.

10. Portillo, I. Baryon susceptibilities from a holographic equation of state. Nucl. Phys. A 2017, 967, 916-919.

11. Portillo, I. Susceptibilities from a black hole engineered EoS with a critical point. J. Phys. Conf. Ser. 2017, 832, 012041.

12. Rougemont, R.; Noronha, J.; Noronha-Hostler, J. Suppression of Baryon Diffusion and Transport in a Baryon Rich Strongly Coupled Quark-Gluon Plasma. Phys. Rev. Lett. 2015, 115, 202301.

13. Rougemont, R.; Ficnar, A.; Finazzo, S.; Noronha, J. Oscillons in a perturbed signum-Gordon model. J. High Energy Phys. 2016, 4, 102.

14. Rougemont, R.; Critelli, R.; Noronha-Hostler, J.; Noronha, J.; Ratti, C. Dynamical versus equilibrium properties of the QCD phase transition: A holographic perspective. Phys. Rev. D 2017, 96, 014032.

15. Finazzo, S.I.; Rougemont, R.; Marrochio, H.; Noronha, J. Hydrodynamic transport coefficients for the non-conformal quark-gluon plasma from holography. J. High Energy Phys. 2015, 2, 51.

(C) 2019 by the authors. Licensee MDPI, Basel, Switzerland. This article is an open access article distributed under the terms and conditions of the Creative Commons Attribution (CC BY) license (http://creativecommons.org/licenses/by/4.0/). 